\title{
Proteomic and immunoassay characterization of a new food allergen from hazelnut (corylus avellana)
}

\author{
G Picariello $^{1 *}$, R Nocerino $^{2}$, C Nitride $^{3}$, G Mamone $^{1}$, R Troncone $^{4}$, P Ferranti $^{1}{ }^{1,3}$, R Berni Canani $^{4}$ \\ From Food Allergy and Anaphylaxis Meeting (FAAM 2013) \\ Nice, France. 7-9 February 2013
}

\section{Background}

Hazelnut (Corylus avellana) is a common cause of lifetime lasting IgE-mediated food allergy. Symptoms range from mild oral allergy syndrome to severe life-threatening anaphylaxis. We aimed to identify allergenic determinants in children living in the Campania region (Italy) with hazelnut allergy.

\section{Methods}

Otherwise healthy children with oral food challenge confirmed hazelnut allergy were prospectively evaluated. Crude protein extracts were obtained from 5 hazelnut varieties, including autochthon, Northern Italy and Oregon (USA) cultivars, with phosphate saline buffer, $\mathrm{pH}$ 7.2. The immunoreactive protein components were identified by SDS-PAGE electrophoresis and Western immunoblotting, using patients sera as source of specific IgE. The IgE-binding protein bands were characterized by advanced proteomic strategies and tandem mass spectrometry (MS)-based de novo peptide sequencing.

\section{Results}

Four subjects were evaluated ( 2 male, $50 \%$; mean age $39 \mathrm{~m}$ ). Symptoms were: urticaria (2), angioedema (3), anaphylaxis (2). No significant differences were observed considering the main demographic and clinical characteristics at diagnosis. All children's sera were immunoreactive to a protein, not previously annotated in database, occurring in hazelnut regardless the variety. The allergen was isolated by combined chromatographic strategies. Only one patient exhibited an additional reactivity to the vicilin-like 7S $48 \mathrm{kDa}$ glycoprotein (Cor a 11). The MS-based characterization provided evidence of a high homology degree between the IgE-binding protein subunit and 11S globulin-like storage proteins expressed in other seeds. The new allergen shares structural traits with the hazelnut $11 \mathrm{~S}$ globulin-like proteins (Cor a 9) such as the disulfide linkage of two subunits, an acidic $(\sim 35 \mathrm{kDa})$ and an alkaline $(\sim 21 \mathrm{kDa})$ one. Interestingly, only the alkaline subunit exhibits antigenic properties.

\section{Conclusion}

A previously unrecognized hazelnut allergen was identified. Except for a faint IgE reactivity of Cor a 11 recorded in a single case, the new allergen was the unique IgE-binding protein in our patients. Future study are warranted to better define possible prognostic and immunotherapeutic implications.

\section{Disclosure of interest}

None declared.

\section{Author details \\ ${ }^{1}$ Institute of Food Sciences - CNR, Avellino, Italy. ${ }^{2}$ Department of Pediatrics, University of Naples "Federico II", Naples, Italy. ${ }^{3}$ Department of Food Science, University of Naples "Federico II", Portici (Naples), Italy. ${ }^{4}$ Department of Pediatrics and European Laboratory for the Investigation of Food Induced Diseases (ELFID), University of Naples "Federico II", Naples, Italy.}

Published: 25 July 2013

doi:10.1186/2045-7022-3-S3-P40

Cite this article as: Picariello et al:: Proteomic and immunoassay characterization of a new food allergen from hazelnut

(corylus avellana). Clinical and Translational Allergy 2013 3(Suppl 3):P40.

${ }^{1}$ Institute of Food Sciences - CNR, Avellino, Italy

Full list of author information is available at the end of the article 